\title{
Estructura del proceso de decisión de compra en los jóvenes universitarios en equipos de telefonía celular: caso de estudio población universitaria en Monterrey Nuevo León (Structure of the buying decision process among college students in cellular phone equipment: case of study on the college student population in Monterrey Nuevo Leon)
}

\author{
Francisco Torres, José N. Barragán, Leticia Neira \& Teresa Pérez \\ UANL, San Nicolás, N.L. México, franciscot@gmail.com
}

Key words: Decision Process, marketing, psychological, situational, social

\begin{abstract}
Influences among college students such as social situational frame, marketing efforts and purchase decision process of cellular phones is the main objectives in this research, which is oriented to analyze the preferences and attitude in shown in the purchase of cellular telephones. The results allows interesting information on how college students consider which is the most innovating manufacturer in cellular telephony, besides how they rank their best provider/ carrier of cellular services and also how they shown their attitude towards the communication channels appropriate for receiving news about technology innovation and sale promotions. The research project was developed among college students from nine universities in the Metropolitan Area of Monterrey (AMM).
\end{abstract}

Palabras Clave: Mercadotecnia, Psicológico, situacional, social, toma de decisiones

Resumen: La influencia en los jóvenes universitarios de los elementos sociales, de mercadotecnia y psicológicos en el proceso de compra de equipos de telefonía celular es el objetivo primordial en esta investigación, la cual es orientada a conocer las preferencias y actitud en la compra de teléfonos celulares. Los resultados arrojan datos interesantes sobre como los jóvenes consideran cual es la marca más innovadora en materia de telefonía celular, además de cómo califican a la mejor prestadora de servicios y su actitud ante la propuesta de comunicación sobre innovación de tecnología y promociones. El proyecto se desarrollo entre estudiantes de nueve universidades del Área Metropolitana de Monterrey (AMM). 


\section{Introducción}

Durante la última década y gracias al avance de la investigación en tecnología de comunicaciones, el teléfono celular se ha convertido en una frecuente herramienta de comunicación, en donde la participación de numerosas compañías prestan servicios a millones de clientes en todo el mundo (Jengchung, Phyo, \& William, 2008).

El éxito de cualquier campaña en mercadotecnia es posible evaluarla cuantitativamente por medio de las utilidades generadas y todo este proceso inicia con el entendimiento fundamental de las necesidades del cliente, y de ésta manera desarrollar estrategias y herramientas aplicables en la campaña de mercadotécnica (Lamb, Hair \& Mc Daniel, 2008; Peter \& Donnelly, 2004). El perfil de los consumidores ofrece diferentes tipos de conducta respecto a cada tipo de producto. Cuando se adquieren artículos de rutina o denominados "bienes de consumo" el cliente generalmente emplea los protocolos ya conocidos, como es el caso de la compra de combustible para el automóvil, productos de limpieza y la mayor parte de estos artículos ya son predefinidos inconscientemente por el consumidor aun antes de llegar al establecimiento de compra, por otro lado cuando se pretende adquirir un producto, que por sus características, su diseño y funcionamiento es cambiante como lo son los artículos electrónicos, automóviles, ropa por mencionar algunos cuantos, en este caso, el individuo se enfrenta a un proceso que lo obliga a la toma de decisiones (Pride, Hughes, \& Kapoor, 2008; Loudon \& Della, 1993)

El proceso de compra consiste de manera general en una estructura sistémica de seis pasos, en donde primero inicia con el reconocimiento del problema de la existencia de una necesidad por obtener determinado producto; segundo, prosigue con la búsqueda de información acerca del producto; tercer paso es la evaluación de las alternativas; cuarto es con base a la evaluación de la información recopilada se lleva a cabo la toma de decisión de compra; quinto es la acción física de compra y, finalmente, el sexto es la evaluación post compra en donde cada vez que interactuamos con el producto, desarrollamos experiencias, y es ahí donde el consumidor encuentra características favorables y desfavorables (Dibb, \& Simkin, 1996; Ferrell, \& Hartline, 2008; Pride, \& Ferrell, 2007)

\section{F. Torres, J.N. Barragán, L. Neira \& T. Pérez}


Los consumidores pueden ser influenciados e incluso persuadidos a comprar un producto por la publicidad a la que han estado expuestos en diferentes modalidades, así también como por las diversas experiencias y factores que ocurren en el proceso de compra (Ferrell, \& Hartline, 2008; Dibb, \& Simkin, 1996). Para entender la forma del por qué los clientes compran en particular las marcas y tipos de teléfonos celulares, se debe tomar en consideración todos los posibles y relevantes procesos que intervienen en la decisión de compra (Jengchung, Phyo, \& William, 2008). Por medio de la investigación en la estructura de la toma de decisiones, no permite comprender que pueden existir diferentes tipos de influencias que llegan a persuadir de manera significativa al cliente potencial y actual. Podemos definir la influencia como el conjunto de elementos que pueden llegar a estar presentes durante el proceso de decisión de compra y que no surgen de un conocimiento pleno o natural de atributos personales y estímulos y puede tener como objetivo el influenciar el conocimiento previo donde el efecto es demostrable y sistemático en el comportamiento del individuo. (Belk, 1974; Dibb, \& Simkin, 1996; Ferrell, \& Hartline, 2008).

En previas investigaciones en ciencias sociales se han percatado del poder que tiene sobre la conducta el entorno social y las interacciones entre individuos (Báez \& Tudela, 2007; Dibb, \& Simkin, 1996; Ferrell, \& Hartline, 2008; Peter \& Donnelly, 2004). Estas investigaciones han llegado a concluir que para el estudio de toma de decisiones de compra es necesario tomar en consideración las influencias de tipo social, de mercadotecnia, situacional y psicológica (Forgas \& Williams, 2001).

\section{Influencia Social}

La influencia social afecta el comportamiento de los individuos, y algunas veces es la normativa de actuación dentro del mismo grupo de personas, la cual pudiera tener efecto en cualquier tiempo, y desde el punto de vista de la investigación en mercadotecnia, es una los factores del patrón de conducta de los consumidores. (Bearden, Calcich, Netemeyer \& Teel, 1986; Engel, Blackwell, \& Miniard, 1990)

La persuasión es uno de los elementos de la influencia social y este reside en la utilización deliberada de los canales de comunicación con el 
propósito de cambiar, modificar o fortalecer las actitudes de los individuos (Forgas \& Williams, 2001; Reardon, 1991).

Existe una necesidad fundamental del humano por pertenecer a grupos o redes sociales, donde este puede pertenecer a más de un grupo a la vez y donde tiene un rol cambiante dentro del mismo grupo. Dentro de estas redes existen reglas formales e informales que influencian el comportamiento de los integrantes de dicha asociación. A lo largo de la vida de un individuo puede formar parte de diferentes grupos sociales (Forgas \& Williams, 2001)

Actualmente los estudiantes universitarios esta obligados a utilizar tecnología que les permitan cumplir con sus obligaciones escolares. Un estudio realizado por investigadores de la Universidad de Houston de departamento Salud y Desarrollo Humano (University of Houston Department of Health and Human Performance, 2008) encontraron que los estudiante que tomaron el curso de la manera "Hibrida" en las cuales se requiere el uso de tecnología a lo largo del curso obtuvieron mejores resultados en promedio en comparación con los alumnos que tomaron la clase de la forma tradicional.

Las nuevas tecnologías están cambiando la forma en que los estudiantes realizan las actividades escolares en donde los estudiantes tienen diferentes estrategias de búsqueda de información, elaboración de tareas y asignaciones, interacción con maestros y compañeros tanto solo por mencionar algunos ejemplos. Dentro de las actividades comunes de los estudiantes de educación superior, según el estudio anterior, encontramos que los estudiantes utilizan en su interacción, plataformas tecnológicas contemporáneas tales como son Google, Twitter, Facebook, Youtube, iTunes, así como a través de los mismos portales educativos de cada universidad (Wetzel, 2010).

Estas prácticas no simplemente son utilizadas por los estudiantes sino que al mismo tiempo son utilizadas por universidades alrededor del mundo como por ejemplo en Estados Unidos en la Universidad de Stanford, que utiliza aplicaciones en iTunes para publicar ensayos de videos sobre clases reales impartidas previamente (Wetzel, 2010). En otras universidades como el caso del MIT (Massachusetts Institute of Technology) en su página ofrece cursos en línea abiertos y accesibles al publico. En Inglaterra, la Open University ofrece la plataforma Open Learn que es un portal educativo para

\section{F. Torres, J.N. Barragán, L. Neira \& T. Pérez}


promover el autoestudio y a su vez el autoaprendizaje. En México la UNAM y UANL ponen a disposición material educativo en sus respectivos sitios de internet así como videos de clases y cursos por medio del portal Youtube. Es posible determinar que las universidades anteriormente mencionadas cuentan con su espacio en el Facebook. Estas herramientas son ejemplos de toda la infraestructura de tecnología y comunicación existente que un estudiante puede utilizar para su desarrollo personal. Otro caso frecuente es cuando un estudiante o profesor de licenciatura o posgrado de la UANL encuentran un curso similar en otra universidad extranjera como la Universidad de Stanford puede libremente opta por descargar las clases en su computadora o celular y poder apreciar las diferencias en el material educativo y las técnicas de enseñanza y aprendizaje.

Por lo anterior, podemos comprobar que el desarrollo de nuevas tecnologías para teléfonos celulares y la demanda en las competencias de educación a nivel superior han permitido darle usos que simplemente antes no habían sido pensados y que van más allá de tan solo realizar y recibir llamadas, enviar mensajes de texto o tomar fotos y videos. Esta nueva utilización de equipos celulares permite el rastreo de información, incorporación y participación en redes sociales, auto aprendizajes en portales educativos, por lo cual es importante para las estructuras sociales y educativas, así como para las mismas empresas, el identificar cuáles son las características principales que buscan los estudiantes al momento de adquirir un teléfono celular, que conlleva de igual forma a una inversión monetaria considerable.

\section{Influencias de la Mercadotecnia}

Los departamentos de mercadotecnia y ejecutivos de publicidad con el objetivo de atraer clientes, frecuentemente emplean estrategias de publicidad con campañas en donde resaltan específicamente las marcas y modelos de teléfonos celulares mencionando las características relevantes de cada equipo. (Hengyuan, Qing, Ligang, \& Guisheng, 2009). Cada estrategia de comercialización puede afectar de diferente forma a los consumidores ya que el producto puede estar fuertemente influenciado por su trayectoria de marca, calidad, innovación, su empaque, información sobre el producto, tipos de publicidad, promociones de ventas, personal de 
ventas, y eventos, por mencionar algunos. (Peter \& Donnelly, 2004; Salmi \& Sharafutdinova, 2008).

El conocimiento sobre el producto que se refiere a la cantidad de información que el consumidor ha llegado a retener en su memoria sobre los diferentes productos, en particular, sobre el diseño atractivo del producto, y relacionarlo con marca, modelo y formas de adquisición. (Lee \& Murphy, 2008).

En estudios recientes, se muestra como la decisión final de compra puede estar influencias fuertemente mediante el contacto físico del consumidor potencial con el producto tangible, especialmente cuando tiene la oportunidad de comprobar los usos y aplicaciones mediante la operación del producto. Esto puede llegar a demostrar que el elemento tangible y visible en el diseño del teléfono celular es decisivo en el momento. Las características como el precio y el servicio también son un elemento tangible de la comercialización ya que en la mayoría de las veces influye considerablemente en la decisión del consumidor cuando se le permite analizar las ventajas competitivas y comparativas del producto y la compañía. (Jengchung, Phyo, \& William, 2008; Ferrell, \& Hartline, 2008).

El comportamiento del consumidor puede ser influenciado en muchos sentidos por la publicidad en donde se reconoce que existe una parte emocional y una cognitiva con respecto al producto y a la marca, tal como son la importancia del status quo y los rangos de distinción social en grupos de referencia. (Biehal, Stephens, \& Curlo, 1992 ; MacKenzie, \& Belch, 1986; Edell, \& Burke, 1987; Meirick 2002). Las empresas conscientes de estas conductas, utilizan estrategias como anuncios de televisión 0 patrocinio de eventos deportivos ya que estos aumentan la probabilidad de que el cliente interactué con elementos de la marca que lleven a influenciar su compra. La publicidad puede influenciar tanto en la compra de nuevos equipos celulares, así como a conservar la lealtad de los clientes hacia la marca y de esta forma blindarlos contra los anuncios de la competencia. (Lee, \& Murphy, 2008; Zarantonello \& Schmitt, 2010).

\section{Influencias Situacionales}

Dentro del proceso de compra se puede llegara a considerar que el elemento situacional es de importancia en la toma de decisión, ya que puede 
lograr alterar la decisión final del consumidor, representando todo un reto para los vendedores de marca (Hawkins, 1994; Peter \& Donnelly, 2004).

Desafortunadamente no se ha encontrado alguna teoria que pudiera definir de manera integral los escenarios situacionales, más sin embargo, investigaciones en el campo de la psicología y mercadotecnia, abordan la influencia situacional como enmarcada desde un enfoque cognitivo conductual, la cual está relacionada con el estudio de los estímulos y respuestas de los individuos. (Hawkins,1994; Dibb, \& Simkin, 1996)

Muchas veces existe un conocimiento previo acerca del producto que se desea comprar, esta información o percepción de los consumidores fue obtenida posiblemente por anuncios publicitarios o recomendaciones de otras personas en donde es posible obtener la información. Resulta por lo tanto, de manera importante el poder captar la atención del consumidor y llegar a ser determinante en la toma de decisiones. En esta parte es vital el llegar a definir el tipo de información que se desea transmitir y las vías de comunicación en donde es más eficiente su envío (Ferrell, \& Hartline, 2008; Pride, \& Ferrell, 2007).

Siendo el principal objetivo en esta investigación, el llegar a conocer las preferencias de los jóvenes universitarios al momento de adquirir teléfonos celulares, resulta pertinente referirnos a las influencias situacionales como las influencias presentes en la selección de compra o de uso de un producto promoviendo el poder encontrar todo lo que pudiera llegar a ser considerado como un elemento situacional que pudiera influencia o motivar al cambio en la manera de pensar sobre algún producto o servicio.

\section{Influencias Psicológicas}

Desde el campo de la psicología el proceso de compra puede será abordado desde diferentes paradigmas, siendo de interés a la temática de esta investigación, resulta relevante el conocer el valor que le otorgan los consumidores a los diferentes atributos internos y externos de un celulares en su proceso de compra. Para lograr esto nos posicionamos en el paradigma cognitivo donde nos explica que para entender la toma decisiones debes tomar en cuenta tres elementos que son: el primero, que es el elemento cognitivo, que es lo que se piensa; el segundo, lo que siente, sus

\section{Decisión de Compra \& Telefonía Celular}


"emociones" y finalmente el operacional, lo que se hace (Báez \& Tudela, 2007; Peter \& Donnelly, 2004).

Analizando el proceso de compra bajo el esquema de celulares decimos que el primer paso se basa en el reconocimiento del problema de donde existe una necesidad del individuo, y aquí podemos ver reflejados los aspectos cognitivos donde el individuo conoce el producto antes adquirirlo y donde puede considerar una emoción de necesidad por obtener un celular. En un segundo paso empieza la búsqueda de información donde el individuo adquiere conocimiento de modelos, marcas y servicios. En un tercer paso es la evaluación de las alternativas dado el conocimiento previo empieza una lucha entre conocimiento y emociones en donde se ve reflejado en juicio en la hora de la compra. En el cuarto es donde es individuo toma la decisión al elegir un producto basado en sus conocimientos y emociones en último paso es la evaluación del producto, y en este caso el individuo una vez que obtuvo el producto e interactúa de forma continua conocerá mas a profundidad las funciones, la calidad e imagen del producto, que lo llevara a la expresión y difusión en su entorno social de su opinión y los demás expresaran su punto de visto donde los comentarios positivos y negativas modificaran la parte cognitiva, emocional y operacional. (Báez \& Tudela, 2007; Dibb, \& Simkin, 1996; Ferrell, \& Hartline, 2008; Peter \& Donnelly, 2004; Pride, \& Ferrell, 2007)

Dentro de cada uno de los pasos del proceso de compra existe una parte cognitiva donde se forman opiniones y se categorizan por medio de las información, un ejemplo es la categorización por marca, precio, diseño, funcionalidad, por lo mencionar algunas. Las cuales con llevan a una respuesta emocional para cada uno de los equipos tales como, duda, confusión, deseabilidad, frustración, alegría, disgusto y rechazo. La evaluación del elemento de razón y emoción permite al individuo operacionalizar y pasar al siguiente 0 retroceder dentro de los pasos del proceso de compra (Báez \& Tudela, 2007; Dibb, \& Simkin, 1996; Ferrell, \& Hartline, 2008; Peter \& Donnelly, 2004; Pride, \& Ferrell, 2007)

La negociación del conocimiento (Jonassen 2000, 2003) marca que es difícil anticipar todas las posibles estrategias operacionales de un individuo en alguna actividad específica, y por lo tanto se debe presentar el conocimiento de tal forma en que se negocia el uso de significados adquiridos a través del contacto con la información e intención de dar una 
respuesta a la situación con la que se enfrenta cada uno. Por tal motivo es importante llegar a identificar los valores de significación que se le dan a los atributos de los celulares dentro de las diferentes categorías (Ferrell, \& Hartline, 2008; Pride, \& Ferrell, 2007).

La ventaja de esta información es el poder obtener las predilecciones del individuo que permiten al mismo llegar a organizar o transformar el material que necesita aprender en su forma más conveniente y los demás con el objetivo de realizar una compra. Se debe tomar en cuenta que en ocasiones las personas pudieran tener una preferencia temporal 0 circunstancial.

\section{Objetivos}

Una vez determinada la importancia del problema de investigación, se determinan los siguientes objetivos

- Estudiar el proceso de toma de decisiones de compra de los estudiantes universitarios en equipos celulares

- Establecer la categorización en cuanto a preferencia de marcas.

- Impacto en la decisión de compra de equipos celulares de las características de diseño, función y servicio en un teléfono celular.

- Análisis de las influencias de tipo social, de mercadotecnia, situacional y psicológica.

\section{Preguntas de Investigación}

¿Cuáles son los factores que influyen en la toma de decisiones de compra de un equipo celular?

¿La influencia social, mercadotecnia, situacional y psicológica pudiera impactar en la toma de decisiones en la compra de un celular?

¿Existe alguna diferencia significativa en la toma de decisiones en la compra de un equipo celular entre hombres y mujeres?

\section{Hipótesis}

Debido a la naturaleza del tipo de investigación exploratoria la cual se fundamenta dentro de una perspectiva cualitativa las dos primeras preguntas 
no permite realiza una hipótesis. Si no más bien aportan una perspectiva de validez de contenido con respecto a los constructos planteados por la tarea.

Por otro lado la tercera pregunta investigación debido a que se basa en una comparación entre género nos permite identificar las formas de pensamiento que pudiera darse entre hombres y mujeres lo cual se presenta en la siguiente hipótesis:

Ho: Hombres= Mujeres

H1: Hombres $\neq$ Mujeres

\section{Metodología}

El diseño de la investigación es de tipo exploratorio y descriptivo, con enfoque multidisciplinario mediante la utilización de metodología cuantitativa que permita analizar la toma de decisiones en el proceso de compra que muestra la población universitaria con respecto a los atributos y características más relevantes según estos perfiles y además del tipo de influencias que afecta la toma de decisiones en compra, y la posible diferenciación entre genero de los participantes.

El instrumento fue elaborado por el autor el cual fue habilitado para su repuesta en línea en el siguiente sitio de Internet: http://www.cienciascognitivas.com. Este diseño tuvo como objetivo el poder medir las preferencias entre los atributos más importantes a evaluar por los participantes en la compra de un equipo celular. También fue importante considerar el poder conocer la percepción de los participantes sobre cual consideran marca más innovadora en el campo de equipos móviles.

\section{Muestra poblacional}

La muestra fue realizada a conveniencia mediante la extensión de invitación abierta, lo que logro una muestra de $n=223$ universitarios de 9 diferentes universidades en donde todos los participantes como condición de aceptación como unidad de estudio, es el poseer un celular propio con el objetivo de garantiza la experiencia en el proceso de compra de equipo celular. Se pretende evaluar los atributos preferidos por los jóvenes universitarios. La información fue analizada utilizando el SPSS para hacer un 
análisis de primera instancia utilizando Frecuencias y ANOVA para diferenciación de grupos.

El equipo de investigadores desea expresar su más sincero agradecimiento a los siguientes maestros y alumnos de las universidades participantes en ese proyecto de investigación, por su labor de coordinación y organización de los grupos estudiantiles:

Mtro. Lic. Amador Garres Rodríguez de la Universidad de Comunicación Avanzada; Mtra. Lic. Denisse González Nery de la Universidad Ateneo de Monterrey; Daniela Góngora Sánchez de la Universidad Regiomontana, José Guadalupe Villarreal Barragán de la Universidad del Norte; Roberto Contreras Cruz de la Universidad Metropolitana de Monterrey, y a todos los estudiantes que participaron activamente de las universidades: Universidad Autónoma de Nuevo Léon, Universidad Tec Milenio, Centro de Estudios Universitarios y Universidad de Monterrey.

\section{Resultados}

La primer parte del estudio consistió en que los participantes calificaron de una lista de características de los equipos celulares cuales de estas consideraban como importantes en donde otorgaba una calificación que reflejaba la ponderación de valor cuantitativo de estas características en los equipos celulares, está lista se integro por 30 atributos que fueron categorizadas en: Diseño, Servicio y Tecnología en donde la puntación más alta fue obtenida por diseño seguida de servicio y tecnología. Al hacer la comparación de grupos mediante Anova F-Test para encontrar diferencias entre hombres y mujeres el valor diseño se observa $0.325 p>0.05$ y para servicio $.328>0.05$ lo que explica que no hay diferencias estadísticamente significativas entre hombres y mujeres con respecto a los atributos de servicio y diseño. Por otro lado podemos observar que los valores de significancia de tecnología 0.00 donde son valores menores a 0.05 observamos que existen valores estadísticamente significativos donde se deduce que los grupos de hombres y mujeres son diferentes.

La segunda parte de estudio tenía el propósito de evaluar cuestiones de preferencias con respecto a marca, servicio, promoción y conductas de compra. 
La primera parte en esta segunda sección del estudio era evaluar la razón se compro el equipo que actualmente poseen en donde las respuestas fueron categorizadas en precio, tecnología y diseño. Ver tabla 1.

Tabla 1. Porcentajes de criterio de preferencias al seleccionar un celular

\begin{tabular}{llll}
\hline & Todos & Hombres & Mujeres \\
\hline Diseño & $22.4 \%$ & $13.3 \%$ & $29.6 \%$ \\
Precio & $44.8 \%$ & $42.9 \%$ & $46.4 \%$ \\
Tecnología & $32.7 \%$ & $43.9 \%$ & $24.0 \%$ \\
\hline
\end{tabular}

Siendo la variable del precio, importante en la decisión de los universitarios en el momento de la compra, y fue importante medir el grado de ponderación en este factor. Ver tabla 2.

Tabla 2. Porcentaje impacto del precio por género

\begin{tabular}{llll}
\hline Periodo & Todos & Hombres & Mujeres \\
\hline Muy Importante & $30.5 \%$ & $33.7 \%$ & $28 \%$ \\
Importante & $50.7 \%$ & $44.9 \%$ & $55.2 \%$ \\
Poco & $15.7 \%$ & $18.4 \%$ & $13.6 \%$ \\
Importante & & & \\
Nada Importante & $3.1 \%$ & $3.1 \%$ & $3.2 \%$ \\
\hline
\end{tabular}

Podemos observar que la importancia del precio en la decisión de compra es muy fuerte tanto para hombres y mujeres ya que el valor de significancia es de .909 el cual es mayor de 0.05 lo que demuestra que no existen diferencias estadísticamente significativa entre la opinión de hombres y mujeres

Cuando se les pidió que se evaluara las compañías existentes en el mercado mexicano (Samsung, Nokia, Motorola, Apple, Blackberry, LG, Palm, Sony Erickson, Sanyo Panasonic). Hubo dos calificaciones la primero refleja la percepción sobre la compañía que mejor innova y el segundo refleja la calificación en general de cada compañía.

La tabla 3 muestra los resultados sobre la percepción de la innovación de las compañías en equipo en celulares

\section{F. Torres, J.N. Barragán, L. Neira \& T. Pérez}


Tabla 3. Percepción de los consumidores con respecto a las compañías por género

\begin{tabular}{llll}
\hline Compañía & Todos & $\begin{array}{l}\text { Percepción } \\
\text { Hombres }\end{array}$ & $\begin{array}{l}\text { Percepción } \\
\text { Mujeres }\end{array}$ \\
\hline Samsung & 4.0 & 1.0 & 6.4 \\
Nokia & 22.9 & 30.6 & 16.8 \\
Motorola & 4.9 & 6.1 & 1.0 \\
Apple & 35.4 & 32.7 & 37.6 \\
Blackberry & 13.5 & 12.2 & 14.4 \\
LG & 4.0 & 4.1 & 4.0 \\
Palm & 1.3 & 2.0 & 0.8 \\
Sony Erickson & 13.5 & 10 & 16.0 \\
Sanyo & - & - & - \\
Panasonic & 0.4 & 1.0 & - \\
\hline
\end{tabular}

Y las calificaciones de cada compañía tomando en cuenta aspecto de diseño, tecnología y funcionalidad fueron las siguientes (Escala 1-5 donde 1 es un rendimiento bajo y 5 rendimiento excelente.) Ver tabla 4.

Tabla 4. Calificación de compañía utilizando el criterio de diseño, tecnología y funcionalidad

\begin{tabular}{lllll}
\hline Compañía & Todos & $\begin{array}{l}\text { Percepción } \\
\text { Hombres }\end{array}$ & $\begin{array}{l}\text { Percepción } \\
\text { Mujeres }\end{array}$ & Sig \\
\hline Samsung & 2.53 & 2.60 & 2.47 & .607 \\
Nokia & 3.11 & 2.98 & 3.21 & .326 \\
Motorola & 2.38 & 2.29 & 2.45 & .491 \\
Apple & 3.42 & 3.58 & 3.30 & .203 \\
Blackberry & 2.78 & 2.81 & 2.75 & .825 \\
LG & 2.67 & 2.60 & 2.73 & .561 \\
Palm & 2.40 & 2.17 & 2.58 & .101 \\
Sony & 2.74 & 3.06 & 2.49 & .030 \\
Erickson & & & & \\
Sanyo & 2.26 & 1.89 & 1.97 & .941 \\
Panasonic & 2.29 & 2.66 & 2.00 & .007 \\
\hline
\end{tabular}


Como observamos en ambas tablas que existe una relación entre la percepción de innovación en el producto como en la calificación general al desempeño de la compañía en diseño, tecnología y funcionalidad dándoles un posicionamiento casi igual entre ambos calificadores. El valor de significancia para Samsung, Nokia, Motorola, Apple, Blackberry, LG, Palm, Sanyo son mayores de 0.05 lo que demuestra que no existen diferencias estadísticamente significativa sobre la opinión de las marcas entre hombres y mujeres. Por otro lado existe una diferencia significativa para las marcas de Sony \& Panasonic en donde existen diferencias de opinión entre hombres y mujeres. Debido aunque existe una obsolescencia en los equipos celulares otro aspecto importante que se considero fue la frecuencia con el que los jóvenes compraban un equipo. Ver tabla 5.

Tabla 5. Frecuencias de compra de equipo por género

\begin{tabular}{llll}
\hline Periodo & Todos & Hombres & Mujeres \\
\hline Más de una vez al año & $5.4 \%$ & $5.1 \%$ & $5.6 \%$ \\
Entre 1.5 a 2 años & $49.8 \%$ & $48.0 \%$ & $51.2 \%$ \\
Entre 2 a 3 años & $27.4 \%$ & $28.0 \%$ & $26.4 \%$ \\
Mas de 3 años & $17.5 \%$ & $18.4 \%$ & $16.8 \%$ \\
\hline
\end{tabular}

Como podemos observar en la grafico el periodo más popular es entre 1.5 a 2 años. El valor de significancia es de .610 el cual es mayor de 0.05 lo que demuestra que no existen diferencias estadísticamente significativa entre el tiempo en el que los hombres y mujeres renuevan su equipo celular. Siendo que cuando se adquiere un celular se debe activar bajo una compañía de telefonía móvil es importante evaluar el servicio que prestan en estas compañías. En México existen 4 compañías importantes en el ramo que son Telcel, Movi Star, lusacel y Nextel las cuales abarca la mayoría del mercado mexicano. Ver tabla 6

Tabla 6. Preferencias con respecto a compañías de telefonía móvil

\begin{tabular}{llll}
\hline & Todos & Hombres & Mujeres \\
\hline Telcel & $64.1 \%$ & $67.3 \%$ & $77 \%$ \\
Movi Star & $22.0 \%$ & $18.4 \%$ & $31 \%$ \\
lusacel & $4.0 \%$ & $6.1 \%$ & $3 \%$ \\
Nextel & $9.9 \%$ & $8.2 \%$ & $14 \%$ \\
\hline
\end{tabular}

F. Torres, J.N. Barragán, L. Neira \& T. Pérez 
Existen diferentes medios de difusión de las promociones las cuales pueden llegar influenciar al consumidor. La evaluación de estos medios de difusión fue como se muestra en la tabla 7.

Tabla 7. Evaluación de medios publicitarios

\begin{tabular}{llll}
\hline & Todos & Hombres & Mujeres \\
\hline Internet & $36.3 \%$ & $46.9 \%$ & $28.0 \%$ \\
Email & $12.1 \%$ & $11.2 \%$ & $12.8 \%$ \\
Mensaje de Texto & $42.6 \%$ & $31.6 \%$ & $51.2 \%$ \\
Llamada Telefónica & $9.0 \%$ & $10.2 \%$ & $8.0 \%$ \\
\hline
\end{tabular}

Y un último aspecto tomado en la investigación fue el hecho averiguar si los consumidores estas dispuesto a dar nuevos uso al celular como lo es el de comprar. Ya que esta función ya ha es actualmente utilizada en países como Japón y Estados Unidos. Ver tabla 8.

Tabla 8. Preferencia de compras por celular

\begin{tabular}{llll}
\hline & Todos & Hombres & Mujeres \\
\hline Mucho & $41.7 \%$ & $40 \%$ & $35.2 \%$ \\
Poco & $11.7 \%$ & $12.2 \%$ & $11.2 \%$ \\
Algo & $33.6 \%$ & $28.6 \%$ & $37.6 \%$ \\
Nada & $13.0 \%$ & $9.2 \%$ & $16.0 \%$ \\
\hline
\end{tabular}

Con respecto a este último punto podemos observar que no existe diferencias significativas entre hombres y mujeres ya que el valor de significancia es de .13 el cual es mayor de 0.05 lo que demuestra que no existen diferencias entre la opinión sobre la compra a través del celular hombres y mujeres.

\section{Discusión de resultados}

A través de análisis de las estrategias de mercadotecnia utilizadas por las compañías de teléfonos celulares para la promoción y venta de equipos (Zhu, Wang, Yan \& Wu, 2009; Peter \& Donnelly, 2004; Salmi \& Sharafutdinova, 2008), en donde puede apreciarse que las características

\section{Decisión de Compra \& Telefonía Celular}


más relevantes resultan ser el diseño, la tecnología y el servicio. Esto deriva que en el diseño de la investigación y la elaboración del instrumento, fueran estas tres características las que llevaran al desarrollo del trabajo de campo, y consecuentemente al análisis de los resultados obtenidos.

Los participantes evaluaron los atributos en equipos celulares con el objetivo de conocer su percepción sobre el nivel de importancia que ellos mismos les otorgan a estos atributos, resultando primeramente una diferencia entre hombres y mujeres en cuanto a la importancia que le atribuyen a cada característica, como por ejemplo, los resultados reflejaron que para la mujeres es más importante el diseño, seguido del servicio, terminando con la tecnología; en cambio a los hombres otorgaron más importancia al diseño, seguido por tecnología y finalmente servicio. Esto permite apreciar el impacto de la influencia psicológica en el proceso de toma de decisiones de compra, ya que estos datos establecen una discriminación entre los atributos de hombres y mujeres basando esta apreciación desde el punto de vista cognoscitivo y operacional (Báez \& Tudela, 2007; Peter \& Donnelly, 2004).

Adicionalmente, el apoyo de este tipo de información a las campañas publicitarias, puede ser de utilidad recomendar a las empresas diferenciar estos atributos cuando se requiera el diseño de campañas de mercadotecnia por género, tomando en consideración las preferencias sobre estos atributos en teléfonos celulares.

En referencia al precio, podemos apreciar que su impacto puede derivar dos vertientes de interpretación, una seria la vertiente situacional y la otra la social. Desde la perspectiva situacional, los resultados muestran que el precio llega ser una variable determinante e incluso más importante que tecnología y diseño al momento de la decisión final de compra (Dibb, \& Simkin, 1996; Ferrell, \& Hartline, 2008). Sobre la influencia de la perspectiva social, el precio representa esquemas de identificación entre los jóvenes universitarios, lo que conlleva a ser un elemento de estratos de nivel socio económico (Forgas \& Williams, 2001).

Un patrón de conducta observado con respecto al procesamiento de compra es que los jóvenes universitarios mayormente buscar cambiar su equipo celular en un promedio de un año y medio y dos años, lo que muestra que es parte de una práctica social de actualización de equipos que demandan los usuarios a las marcas de teléfonos.

\section{F. Torres, J.N. Barragán, L. Neira \& T. Pérez}


En este estudio se sometieron a evaluación por los participantes los equipos de telefonía celular que ofrecen actualmente en México, diez marcas de teléfonos. El constructo a evaluar de manera cognitiva fue la percepción sobre la cual consideran al fabricante con la mejor innovación en productos, tecnología, diseño y servicios. Resultando que la marca Apple es la mejor posicionada en la mente de los usuarios universitarios como el fabricante de mejor innovación en estos atributos. A este fabricante, según los resultados le sigue de cerca Nokia en segundo lugar como los más frecuentemente evaluados. Además de la respuesta cognitiva, fue importante la evaluación individual de cada una de las marcas con el objetivo de otorgar una posición a cada una y así poder observar la evaluación cognitiva de cada marca por cada participante. Es importante hacer notar que bajo la perspectiva social, esta marca muestra mayor aceptación entre los jóvenes universitarios.

De conformidad a las estadísticas que presenta la Comisión Federal de Telecomunicaciones en México (Cofetel) donde se muestra un crecimiento en los usuarios de telefonía celular; el incremento es de 59.4 millones a 71 millones de Marzo del 2007, a Marzo de 2010. Esto representa según este organismo una proporción de usuarios del $66 \%$ en telefonía celular.

Según Cofetel, la compañía líder es Telcel, del grupo empresarial América Móvil, en donde su participación en el mercado es $72.5 \%$. Telefónica Movistar con $18.7 \%$ y finalmente lusacell y Nextel muestran solo una participación minoritaria.

Al efectuar una comparativa con los resultados obtenidos, es posible ver un homogeneidad entre las posiciones de estas empresas según la Cofetel, y la percepción de los universitarios es similar en cuanto a que la marca mas mencionada como la mejor servicio es Telcel, seguida por Movistar, en donde lusacel y Nextel muestran participación minoritaria.

Sobre los beneficios que otorgan estas empresas a sus clientes, como promociones por ser clientes de la compañía y la disponibilidad de comunicación ágil entre su entorno social, es posible observar que la mercadotecnia influye como referencia social al momento de la toma de decisión sobre la elegir al prestador de servicios celulares.

Tomando en consideración el importancia del canal de comunicación para captar la atención de los clientes y de esta forma asegurase de la recepción y comprensión del mensaje publicitario, los resultados muestran que los mensajes de texto (SMS) resultan el canal mas atractivo y 
conveniente para los jóvenes para recibir publicidad y promociones, seguido del Internet.

La innovación continúa siendo parte de los esfuerzos más importantes de los fabricantes de equipos celulares, siendo su prioridad la búsqueda de nuevos servicios al usuario, como son la adquisición de productos y servicios a través del teléfono celular, podemos apreciar que en los jóvenes universitarios existe una aceptación por este tipo de servicios. Cabe mencionar que una evaluación anterior por uno de los autores en una muestra de 300 estudiantes de licenciatura en Septiembre del 2005, se encontró un rechazo casi absoluto hacia ese tipo de servicios, lo que contraste con los resultados en esta investigación en donde los jóvenes en cinco años han modificado sus actitudes sobre esta modalidad de compra.

\section{Conclusiones y recomendaciones}

De los hallazgos mas interesantes derivados de esta investigación, se encuentra el procesamiento cognitivo en la toma de decisiones en donde se tomaron en cuenta las influencias en el proceso de compra, las preferencias sobre atributos, características y marcas, resultando diferencias significativas en algunos aspectos, por lo cual es pertinente señalar los aspectos y diferencias relevantes.

Esta investigación permitió apreciar estadísticamente el impacto que tienen el diseño, servicio y tecnología como los atributos a evaluar el momento de adquirir equipo celular y en la selección de la empresa prestadora de servicios por los jóvenes universitarios.

En cuanto a las decisiones por género, el estudio nos muestra que no existen diferencias entre hombres y mujeres no fueron estadísticamente significativas en la mayoría de las variables, simplemente muestran una diferencia en opinión con respecto a la tecnología.

Otro aspecto relevante, con respecto a investigaciones anteriores, es que existe una nueva actitud por parte de los jóvenes a mostrar su disponibilidad para realizar compras por medio del teléfono celular, a diferencia de hace cinco años, que mostraban renuencia antes este sistema de ventas por tecnología móvil. 
Es recomendable para futuras investigaciones que se pueda realizar este mismo estudio con otra población de usuarios distinta a los universitarios de la AMM en donde se posible encontrar diferencias culturales y de apreciación en otra muestra de población. Así mismo, es recomendable este estudio orientado a conocer el apego emocional a marcas, que se traduce en la verdadera lealtad de marca, mas ahora cuando la empresas comienzan a utilizar la "mercadotecnia emocional".

\section{Referencias}

Báez J. \& Tudela P., 2007. Investigación cualitativa. España: ESIC Editorial.

Bearden, W.O., Calcich, S.E., Netemeyer, R., \& Teel, J.E., 1986. An Exploratory Investigation of Consumer Innovativeness and Interpersonal Influence Advances in Consumer Research, 13 (1), 77-82.

Belk, R. W., 1974. An exploratory assessment of situational effects in buyer behavior. Journal of Marketing Research, 11, 156-163.

Biehal, G., Stephens, D. and Curlo, E., 1992. Attitude toward the ad and brand choice Journal of Advertising Research , 21(3), 19-36.

Cofetel, 2008, Mayo 5. Nuevas estadísticas telefónicas en México. Retrieved April 7, 2010 from http://vivirmexico.com/2008/05/nuevas-estadisticas-telefonicas-en-mexico

Dibb, S. \& Simkin L., 1996. The market segmentation workbook: target marketing for marketing managers London: Thomson.

Edell , J . A . \& Burke, M. C., 1987. The power of feelings in understanding advertising effects Journal of Consumer Research, 14 (3), 421-433.

Engel, J. F., Blackwell, R. D., and Miniard, P.W., 1990. Consumer Behavior Orlando, Fla: Dryden Press.

Ferrell, O. C. \& Hartline, M. D., 2008. Marketing Strategy Mason, Oh: Thomson.

Forgas, J.P. \& Williams K.D., 2001. Social Influence: direct and indirect processes Philadelphia, PA: Psychology Press.

Gounaris, S. \& Stathakopoulos, V., 2004. Antecedentsand Consequences of Brand Loyalty: An Empirical Study. Journal of Brand Management , 11(4), 283 -306.

Hawkins, B. (1994). Comportamiento del consumidor (5ta. Ed.) Mexico: Prentice Hall.

Jengchung V., Phyo L. \& William R., 2008. The customer journey when purchasing a new mobile phone: Testing a dual mediation model Direct, Data and Digital Marketing Practice, 10 (2), 116-132.

Jonassen, D.H., 2002. Engaging and supporting problem solving in online learning. Quarterly Review of Distance Education, 3 (1), 1-13.

Jonassen, D.H., 2000. Toward a design theory of problem solving. Educational Technology: Research \& Development, 48 (4), 63-85.

Kim, Y.A \& Srivastava, J., 2007. Impact of Social Influence in E-Commerce Decision Making ACM International Conference Proceeding Series, 258, 293-302.

Decisión de Compra \& Telefonía Celular 
Lamb, C. , Hair, J, \& McDaniel, C., 2008. Essentials of Marketing 6th Edition Mason, OH: South Western .

Lee R. \& Murphy J. A., 2008. The Moderating Influence of Enjoyment on Customer Loyalty Marketing Journal , 16(2), .

Loudon A. \& Della B. D., 1993. Consumer Behavior : Concepts and Applications New York: McGraw Hill.

MacKenzie , S . B . Lutz , R . J . and Belch , G . E ., 1986. The role of attitude toward the ad as a mediator of advertising effectiveness: A test of competing explanations Journal of Marketing, 23, 130-143.

Meirick , P., 2002. Cognitive responses to negative and comparative political advertising Journal of Advertising Research , 31(1), 49-73.

Peter, P. \& Donnelly, J., 2004. Marketing Management: Knowledge and Skills. Cornell: McGraw-Hill Education .

Pride, W. M. \& Ferrell O. C., 2007. Foundations of Marketing Boston, MA: Houghton Mifflin Company.

Pride,W.M., Hughes, R.J. \& Kapoor, J.R., 2008. Business 9th Edition Boston, MA: Houghton Mifflin Company.

Reardon, K.K., 1991. La persuacion en la comunicacion. Teoria y Contexto $\left(2^{a}\right.$ ED.) Barcelona: Paidos.

Salmi, A. \& Sharafutdinova E., 2008. Culture and design in emerging markets: the case of mobile phones in Russia Journal of Business \& Industrial Marketing, 23(6), 384394.

Solomon, M. R., 1997. Comportamiento del consumidor (3ra. Ed.). Mexico: Prentice Hall.

University of Houston, 2008, March 25. College Students Score Higher In Classes That Incorporate Instructional Technology Than In Traditional Classes. ScienceDaily. Retrieved April 6, 2010, from http://www.sciencedaily.com /releases/2008/03/080324125154.htm

Wetzel, D.R., 2010, Feb 25. New Technologies Changing College Education. Adults Students Demand Colleges Keep up With thw Latest Technology. Retrieved April 7, 2010 from http://adult-education.suite101.com/article.cfm/new-technologies-changingcollege-education

Yang, Z., \& Peterson, R. T., 2004. Customer Perceived Value, Satisfaction, and Loyalty: The Role of Switching Costs. Psychology and Marketing , 21 (10), 799-822.

Zarantonello \& Bernd H Schmitt, 2010. Using the brand experience scale to profile consumers and predict consumer behaviour. Journal of Brand Management Online publication date: 26-Mar-2010.

Zhu, H., Wang, Q., Yan,L. \& Wu, G., 2009. Are consumers what they consume? Linking lifestyle segmentation to product attributes: an exploratory study of the Chinese mobile phone market Journal of Marketing Management, 25, 295-314.

\section{F. Torres, J.N. Barragán, L. Neira \& T. Pérez}

